\title{
Fatores associados ao uso de serviços odontológicos por crianças de cinco anos em municípios de pequeno porte populacional
}

Factors associated with use of dental services for children five years in small cities Factores asociados al uso de servicios odontológicos por niños de cinco años en municipios de pequeño porte poblacional

\author{
Maria Paula RANDO-MEIRELLES ${ }^{(1)}$ \\ Vinícius Aguiar LAGES (2) \\ Marília Jesus BATISTA ${ }^{(3)}$ \\ Silvia CYPRIANO ${ }^{(4)}$ \\ Maria da Luz Rosário de SOUSA ${ }^{(5)}$
}

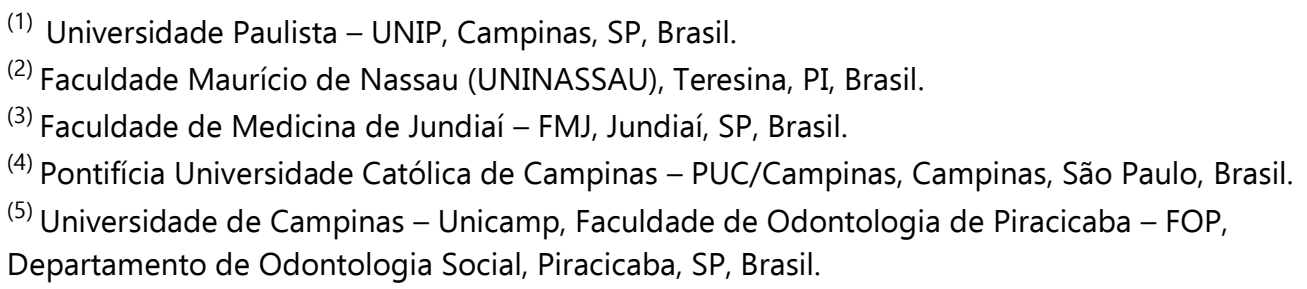

Recebido: 31 jul 2017 Revisad o: 13 jan 2018 Aceito: 15 jan 2018

Autor de correspondência: Vinícius Aguiar Lages viniciusthe@hotmail.com

Conflito de interesses: Os autores declaram não haver nenhum interesse profissional ou pessoal que possa gerar conflito de interesses em relação a este manuscrito.

\begin{abstract}
Resumo
Identificar fatores associados ao uso de serviços odontológicos por 271 crianças de cinco anos de três municípios de pequeno porte do Estado de São Paulo. Os exames odontológicos foram realizados por cinco examinadores, com concordância interexaminadores igual ou superior a 0,65 (Kappa), e seguiram a metodologia proposta pela Organização Mundial da Saúde. O SiC Index foi calculado. Para a análise estatística utilizou-se regressão de Poisson, na qual variáveis que apresentaram valor de $p$ menor ou igual a 0,25 entraram no modelo final da análise multivariada. A variável dependente para as análises do município de Lindóia foi o componente restaurado e para os municípios Águas de Lindóia e Monte Alegre do Sul foi o componente cariado. A variável "ter ido ao dentista" esteve associada tanto ao componente restaurado no município de Lindóia $(p=0,002)$ quanto ao componente cariado nos outros dois municípios $(p=0,00)$, enquanto a variável "escolaridade materna" apenas ao componente restaurado em Lindóia $(p=0,01)$. Concluiu-se que ter realizado tratamento odontológico ou não nesta população de cinco anos esteve associado à procura dos serviços de saúde, sendo que o grau de escolaridade da mãe pode influenciar na saúde bucal dos filhos. O estabelecimento de políticas públicas de promoção de saúde que possibilitem o atendimento às crianças de pouca idade nestes municípios é necessário.
\end{abstract}

Descritores: Saúde Bucal; Cárie Dentária; Epidemiologia; Distribuição de Poisson, Índice CPOD. 


\begin{abstract}
Identify factors associated with use of dental services by 271 children under five three small municipalities of São Paulo State. Dental examinations were performed for five examiners, with interrater agreement of not less than 0.65 (Kappa), and followed the methodology proposed by the World Health Organization. The SIC Index was calculated. Statistical analysis was performed using Poisson regression, in which variables with $p$-value less than or equal to 0.25 into the final model of multivariate analysis. The dependent variable for Lindóia county analysis was restored component and municipalities Lindóia Water and Monte Alegre do Sul was the decayed component. The variable "have gone to the dentist" was linked both to the restored component in the municipality of Lindóia $(p=0,002)$ as the decayed component in the other two municipalities $(p=0,00)$ and the variable maternal education only to the restored component in Lindóia $(p=0,01)$. It was concluded that having had dental treatment or not in this population of five years was associated with the demand of the health services, and the degree of education of the mother can influence the oral health of the children. The establishment of public health promotion policies that enable the care of the young children in these municipalities is necessary.
\end{abstract}

Keywords: Oral Health; Dental Caries; Epidemiology; Poisson Distribution; DMFT Index.

\begin{abstract}
Resumen
Identificar factores asociados al uso de servicios odontológicos por 271 niños de cinco años de tres municipios de pequeño porte del Estado de São Paulo. Los exámenes odontológicos fueron realizados por cinco examinadores, con concordancia interexaminadores igual o superior a 0,65 (Kappa), y siguió la metodología propuesta por la Organización Mundial de la Salud. El SiC Index fue calculado. Para el análisis estadístico se utilizó regresión de Poisson, en la cual variables que presentaron valor de p menor o igual a 0,25 entraron en el modelo final del análisis multivariada. La variable dependiente para los análisis del municipio de Lindóia fue el componente restaurado y para los municipios Aguas de Lindóia y Monte Alegre do Sul fue el componente cariado. La variable "haber ido al dentista" estuvo asociada tanto al componente restaurado en el municipio de Lindóia $(p=$ $0,002)$ en cuanto al componente cariado en los otros dos municipios $(p=0,00)$, mientras que la variable "escolaridad materna" componente restaurado en Lindóia $(p=0,01)$. Se concluyó que haber realizado tratamiento odontológico o no en esta población de cinco años estuvo asociado a la demanda de los servicios de salud, siendo que el grado de escolaridad de la madre puede influenciar en la salud bucal de los hijos. El establecimiento de políticas públicas de promoción de salud que posibiliten la atención a los niños de poca edad en estos municipios es necesario.
\end{abstract}

Palabras-claves: Salud Bucal; Caries Dentales, Epidemiología, Distribución de Poisson; Índice CPOD.

\title{
Introdução
}

A compreensão dos resultados de estudos epidemiológicos em crianças é importante para a identificação dos fatores causais e para o planejamento de medidas que propiciarão a redução da prevalência de cárie no futuro. ${ }^{1,2}$ As ações de promoção de saúde realizadas em âmbitos nacional e local vêm alcançando o objetivo de reduzir a carga da doença, o que pode ser comprovado pelos dados dos levantamentos nacionais de saúde bucal no Brasil. 3,4 Além disso, estudos tem demonstrado maior acesso da população aos serviços 
odontológicos por meio de programas governamentais como Estratégia de Saúde da Família e Brasil Sorridente nas últimas décadas. ${ }^{5,6}$ No entanto, segundo dados da Pesquisa Nacional por Amostra de Domicílios - PNAD de 2010, 77,9\% das crianças de até quatro anos nunca visitaram o dentista. ${ }^{7}$

O uso de serviços de saúde depende da percepção da doença por parte dos indivíduos ou de seus responsáveis, ou seja, reflete, entre outros aspectos, o entendimento que eles têm sobre saúde. ${ }^{8}$ No Brasil, já se registrou que o acesso aos serviços odontológicos, além de ser limitado nos primeiros anos de vida, é desigual e a taxa de procura ainda é baixa, apesar do aumento observado nos anos recentes. ${ }^{5,6}$ As informações sobre o uso de serviços odontológicos, bem como os fatores a eles associados, são importantes na organização das políticas públicas de saúde na atenção primária, fundamentando uma prestação de serviço contextualizada à necessidade real da população. ${ }^{5,8}$

Estudos com crianças na faixa etária de cinco anos demonstraram que famílias de baixa renda, mães com baixa escolaridade e a dificuldade de acesso aos serviços de saúde bucal foram associados à pior condição de saúde bucal..$^{8-12}$ No entanto, o uso dos serviços de saúde não deve ser entendido apenas como a procura e execução do tratamento, pois a necessidade e o comportamento individual diante dos seus problemas de saúde também merecem $_{\text {atenção. }}{ }^{12} \mathrm{O}$ comportamento das crianças, particularmente, está ligado ao responsável pela mesma, ${ }^{13}$ o que desperta maior atenção nesta faixa etária.

O uso da epidemiologia no planejamento de ações e serviços de saúde bucal no Brasil, especialmente na atenção primária, vem aumentando. ${ }^{3,4}$ Entretanto, são raros os estudos que pesquisem municípios de pequeno porte populacional e com a faixa etária de cinco anos. Assim, o objetivo deste estudo foi identificar fatores associados ao uso de serviços odontológicos por crianças de cinco anos de idade em três municípios brasileiros de pequeno porte populacional. 


\section{Metodologia}

O delineamento deste estudo foi do tipo transversal e realizado com crianças de cinco anos, em três municípios de pequeno porte populacional do Estado de São Paulo: Lindóia, Águas de Lindóia e Monte Alegre do Sul. Os três municípios estudados fazem parte da VII Direção Regional de Saúde de São Paulo e participaram do inquérito epidemiológico intitulado "Condições de Saúde Bucal em Municípios localizados na região de Campinas" realizado pela Secretaria de Saúde do Estado de São Paulo. Esta pesquisa foi aprovada pelo Comitê de Ética em Pesquisa da Faculdade de Odontologia de Piracicaba - FOP/UNICAMP, com protocolo 105/2010. Aos pais dos alunos selecionados para participarem do levantamento foi enviado um Termo de Consentimento Livre e Esclarecido - TCLE para assinatura, mediante concordância.

Os escolares avaliados são provenientes de escolas públicas sorteadas destas cidades, que foram classificadas segundo seu porte populacional a partir de critérios do Instituto Brasileiro de Geografia e Estatística - IBGE, que considera de pequeno porte as cidades com até 50.000 habitantes. ${ }^{14}$ Quanto ao tamanho da população e fluoretação da água, em 2010, Lindóia tinha 6712 habitantes e há cinco anos a água de abastecimento pública era fluoretada, Monte Alegre do Sul tinha 7150 habitantes e sete anos com água fluoretada e, Águas de Lindóia, 17266 habitantes e 30 anos com fluoretação. ${ }^{15}$

A amostragem foi sistemática probabilística, de modo a ser representativa para cada um dos três municípios. A seleção da amostra nos municípios ocorreu em dois estágios, sendo que no primeiro estágio as Unidades Primárias Amostrais - UPA foram as escolas e no segundo estágio foram os escolares. Assim, foram sorteadas 20 escolas em cada município, que foram organizadas sistematicamente de acordo com o número de alunos, e posteriormente foram selecionados os alunos de cinco anos. Na realização dos exames adotou-se a regra de não reposição dos elementos amostrais perdidos. O cálculo amostral para cada município, que compôs esta amostra está de acordo com dados das Condições de Saúde Bucal do Estado de São Paulo (2002). ${ }^{16}$ Adotou-se nível de confiança de 95\%, 
precisão de $20 \%$ e efeito do desenho (deff) de 2 . Foram selecionadas todas as crianças de cinco anos matriculadas nas escolas públicas dos três municípios: 88 em Lindóia, 71 em Monte Alegre do Sul e 187 em Águas de Lindóia, totalizando 346.

Os dados foram coletados por meio de exames clínicos e questionário estruturado. Os questionários foram enviados para os pais junto com o TCLE. Cinco examinadores com experiência em levantamentos epidemiológicos participaram do estudo. A calibração dos mesmos ocorreu em oficinas de treinamento que tiveram duração de 32 horas entre conteúdo teórico e prático. Para a padronização da equipe, utilizou-se a técnica do consenso. Tomou-se como referência o modelo proposto pela Organização Mundial de Saúde $(\mathrm{OMS}){ }^{17}$ tendo sido aprovados para participação no levantamento apenas os examinadores que obtiveram coeficiente Kappa para a concordância interexaminadores igual ou superior a 0,65, sendo que o coeficiente Kappa variou de 0,91 a 1 . A concordância intraexaminador variou de 85 a 89,9\%.

Os exames odontológicos seguiram a metodologia proposta pela OMS. ${ }^{17}$ Utilizaramse espelho bucal plano e sonda milimetrada de ponta esférica, sob luz natural, com o examinador e a criança examinada sentados. A experiência de cárie foi avaliada pelo índice de dentes decíduos com lesões de cárie (componente c), perdidos (componente e) e restaurados (componente o), denominado de ceod, e que é calculado a partir do somatório do número de dentes que apresentam estas condições. Os valores deste índice correspondem aos seguintes graus de severidade: muito baixo $(0,0$ a 1,1$)$, baixo $(1,2$ a 2,6$)$, moderado (2,7 a 4,4), alto $(4,5$ a 6,5$)$ e muito alto (a partir de 6,6). Os dados foram registrados por anotadores previamente treinados, em fichas individuais. O índice SIC (Significant Caries Index) foi empregado para definir a severidade da cárie no terço do grupo que apresentou maior experiência da doença. O cálculo da média do ceod e dos componentes cariados, extraídos e restaurados foi realizado no grupo todo (grupo ceod). Além disso, a amostra foi dividida em dois grupos para as mesmas análises: o primeiro com o terço dos indivíduos com os maiores índices de cárie, grupo SIC (alta experiência de cárie), e o outro com os 
demais indivíduos com os menores índices (baixa experiência de cárie). No momento do exame também foram verificadas as necessidades de tratamento odontológico, segundo os critérios propostos pela OMS. $^{17}$

A variável dependente para as análises do município de Lindóia foi o componente restaurado e para os municípios Águas de Lindóia e Monte Alegre do Sul foi o componente cariado. Os dados dos dois últimos municípios foram unidos e analisados em conjunto. Como variáveis independentes, foram incluídas no estudo as sociodemográficas e comportamentais relacionadas às crianças. A decisão de analisar em conjunto os dados dos municípios Águas de Lindóia e Monte Alegre do Sul se deve ao fato de que ambos apresentaram comportamento semelhante quanto ao componente cariado do índice ceod. Nestes municípios, este componente representou mais de $60 \%$ do índice ceod, diferente do município de Lindóia no qual o componente restaurado representou mais de $70 \%$. É importante ressaltar essa diferença entre os componentes, pois ao se avaliar a média do ceod nota-se que a variação entre os três municípios foi de 0,61.

As variáveis sociodemográficas foram: sexo da criança (masculino/feminino), cor da pele (branca/não-branca), quem era o responsável pelo sustento da família (pai e mãe/apenas o pai ou apenas a mãe); anos de escolaridade do pai e anos de escolaridade da mãe (ensino fundamental incompleto/fundamental completo ou mais); número de residentes por cômodo da moradia (até duas pessoas por cômodo/ mais que duas pessoas).

As variáveis comportamentais relacionadas à criança foram: ter faltado à escola devido à dor de dente (sim/não); ter ido ao dentista alguma vez na vida (sim/não); motivo pelo qual foi ao dentista (rotina/dor/tratamento), sendo que em rotina incluiu as respostas: nunca ter ido ao dentista, ter ido por rotina, manutenção e outros; tempo da última visita ao dentista (até dois anos/dois anos ou mais/nunca foi); local da consulta (serviço público/particular ou outros); satisfação com o tratamento recebido (ruim e regular/bom e ótimo); renda familiar no mês anterior à pesquisa (até dois salários-mínimos/mais que dois salários). 
Para a análise dos resultados foi utilizado o software Statistical Package for the Social Sciences - SPSS. Foram realizadas análises estatísticas descritivas das variáveis. Para a análise bivariada, o cálculo para o município de Lindóia foi realizado entre o componente restaurado do índice ceod e as variáveis estudadas, e para os municípios Águas de Lindóia e Monte Alegre do Sul, o cálculo foi realizado entre o componente cariado e as mesmas variáveis utilizadas para o cálculo de Lindóia. As variáveis que apresentaram valor de p menor ou igual a 0,25 na análise bivariada entraram no modelo final da análise multivariada de Poisson.

\section{Resultados}

A amostra total examinada foi de 271 crianças: 88 em Lindóia, 58 em Monte Alegre do Sul e 125 em Águas de Lindóia. As crianças cujos pais não assinaram termo de consentimento ou não estavam presentes no dia do exame nas escolas foram excluídas, o que explica a redução da amostra selecionada previamente.

O município de Lindóia apresentou a maior média de ceod e SIC entre os três municípios e 40,9\% de crianças livres de cárie. Águas de Lindóia demonstrou menor experiência de cárie em ambos os grupos e maior porcentagem de livres de cárie (49,6\%). Monte Alegre do Sul apresentou 36,2\% de livres de cárie. E ao comparar o grupo de alta experiência de cárie - SIC com o grupo como um todo (ceod) nos três municípios, o SIC acompanhou a tendência do índice ceod, ou seja, o município com maior média de ceod tinha também maior média do SIC.

Mais de 70\% dos dentes com experiência de cárie em Lindóia estavam restaurados, ao contrário de Águas de Lindóia que apresentou 87\% de dentes com lesões de cárie no grupo como um todo. Monte Alegre do Sul também apresentou a maioria dos dentes com experiência de cárie com necessidade de tratamento restaurador. Neste último município a necessidade de tratamento restaurador foi semelhante entre os grupos ceod, baixa e alta experiência de cárie, sendo que todos apresentaram maior porcentagem do componente 
cariado. Nos três municípios, a porcentagem dos componentes do grupo de alta experiência (SIC) revelou o mesmo comportamento que o grupo como um todo (Gráfico 1).

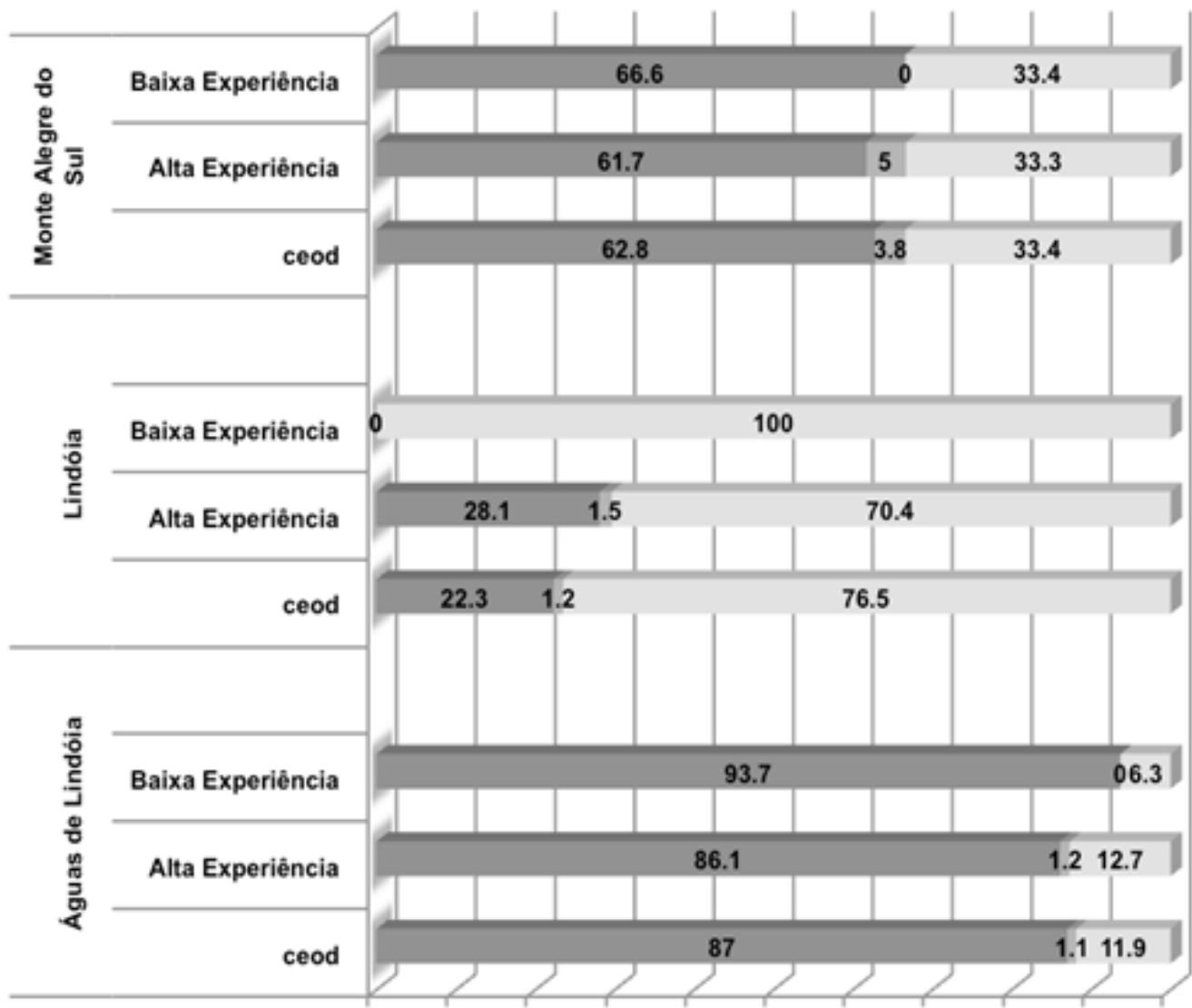

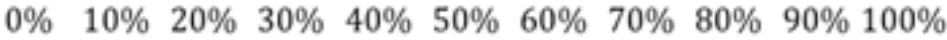

Cariados

Extraídos

Restaurados

Gráfico 1. Porcentagem dos componentes cariado, extraído e restaurado nos municípios de Monte Alegre do Sul, Lindóia e Águas de Lindóia, SP, 2010

Fonte: Elaboração própria (2010).

As Tabelas 1 e 2 demonstram o resultado da análise bivariada. As variáveis associadas ao componente restaurado no município de Lindóia foram: a criança ter ido ao dentista pelo menos uma vez na vida e a mãe ter baixo nível de escolaridade (não ter completado o ensino fundamental). As variáveis associadas ao componente cariado nos municípios Águas de Lindóia e Monte Alegre do Sul foram: a criança não ter ido ao dentista pelo menos uma vez na vida, e quando foi, o motivo da consulta ter sido para consulta de rotina. Na Tabela 3, encontra-se o resultado da análise de regressão de Poisson. 
Tabela 1. Características demográficas, socioeconômicas e comportamentais conforme a presença ou não do componente restaurado em crianças de 5 anos. Lindóia, 2010.

\begin{tabular}{|c|c|c|c|c|c|c|c|}
\hline \multirow{3}{*}{ VARIÁVEL } & & & \multicolumn{5}{|c|}{ COMPONENTE RESTAURADO } \\
\hline & \multicolumn{2}{|c|}{ Total } & \multicolumn{2}{|c|}{ Sadio } & \multicolumn{2}{|c|}{ Restaurado } & \multirow[b]{2}{*}{ p } \\
\hline & n & $\%$ & $\mathbf{n}$ & $\%$ & $\mathbf{n}$ & $\%$ & \\
\hline \multicolumn{8}{|l|}{ Sexo } \\
\hline Masculino & 42 & 47,4 & 13 & 30,9 & 29 & 69,1 & 0,016 \\
\hline Feminino & 46 & 52,6 & 26 & 56,5 & 20 & 43,5 & \\
\hline \multicolumn{8}{|c|}{ Responsável pelo sustento } \\
\hline Pai e mãe & 43 & 48,9 & 21 & 48,8 & 22 & 51,2 & 0,40 \\
\hline Pai ou mãe/outros & 45 & 51,1 & 18 & 40 & 27 & 60 & \\
\hline
\end{tabular}

Escolaridade do pai

$\begin{array}{llllllll}\text { Até ensino fundamental } & 62 & 70,5 & 29 & 46,8 & 33 & 53,2 & 0,47 \\ \text { Fundamental completo ou }+ & 26 & 29,5 & 10 & 38,5 & 16 & 61,5 & \end{array}$

Escolaridade da mãe

$\begin{array}{lccccccc}\text { Até ensino fundamental } & 65 & 73,9 & 32 & 49,2 & 33 & 50,8 & 0,12 \\ \text { Fundamental completo ou }+ & 23 & 26,1 & 7 & 30,4 & 16 & 69,6 & \end{array}$

Faltou à escola por dor

$\begin{array}{lccccccc}\text { Não } & 84 & 100 & 36 & 42,9 & 48 & 57,1 & N V^{*} \\ \text { Sim } & 0 & 0 & 0 & 0 & 0 & 0 & \\ \begin{array}{l}\text { Foi ao dentista alguma vez } \\ \text { Sim }\end{array} & 54 & 65,1 & 14 & 25,9 & 40 & 70,1 & <0,000 \\ \text { Não } & 29 & 34,9 & 0 & 0 & 29 & 100 & \end{array}$

Há quanto tempo foi ao dentista

$\begin{array}{lccccccc}\text { Até } 2 \text { anos } & 55 & 71,4 & 18 & 32,7 & 37 & 67,3 & 0,10 \\ 2 \text { anos ou }+ & 6 & 7,8 & 2 & 33,3 & 4 & 66,4 & \\ \text { Nunca foi } & 16 & 20,8 & 12 & 75 & 4 & 25 & \end{array}$

Onde foi ao dentista

$\begin{array}{lccccccc}\text { Particular ou outros } & 29 & 41,4 & 7 & 24,1 & 22 & 75,9 & 0,89 \\ \text { Público } & 41 & 58,6 & 18 & 43,9 & 23 & 56,1 & \end{array}$

Satisfação com o atendimento

$\begin{array}{llllllll}\text { Bom/ótimo } & 49 & 55,7 & 12 & 24,5 & 37 & 75,5 & <0,000 \\ \text { Ruim/regular } & 39 & 44,3 & 27 & 69,2 & 12 & 30,8 & \end{array}$

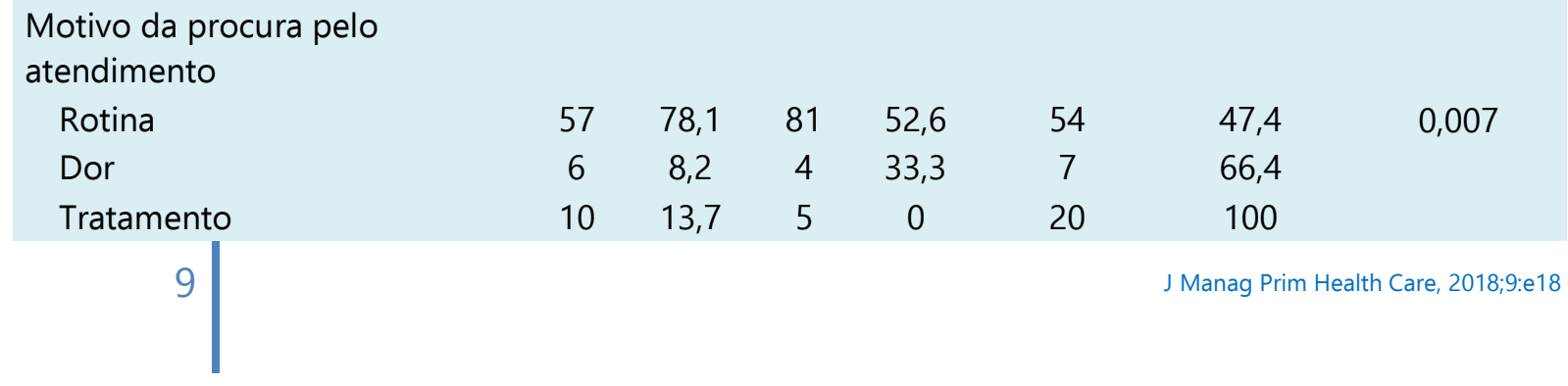


Renda familiar

$\begin{array}{lccccccc}\text { Até } 2 \text { salários-mínimos } & 2 & 2,8 & 0 & 0 & 2 & 100 & 0,23 \\ \text { + que 2 salários-mínimos } & 69 & 97,2 & 29 & 42 & 40 & 58 & \end{array}$

Fonte: Elaboração própria (2010).

* Nenhuma criança faltou a escola por dor de dente.

Tabela 2. Características demográficas, socioeconômicas e comportamentais conforme a presença ou não do componente cariado em crianças de 5 anos. Águas de Lindóia/Monte Alegre do Sul, 2010.

\begin{tabular}{|c|c|c|c|c|c|c|c|}
\hline \multirow{3}{*}{ VARIÁVEL } & \multicolumn{7}{|c|}{ COMPONENTE CARIADO } \\
\hline & \multicolumn{2}{|c|}{ Total } & \multicolumn{2}{|c|}{ Sadio } & \multicolumn{2}{|c|}{ Cariado } & \\
\hline & $\mathbf{n}$ & $\%$ & $\mathbf{n}$ & $\%$ & $\mathbf{n}$ & $\%$ & \\
\hline
\end{tabular}

Sexo

$\begin{array}{lccccccc}\text { Masculino } & 93 & 50,8 & 79 & 84,9 & 14 & 15,1 & 0,6 \\ \text { Feminino } & 90 & 49,1 & 72 & 80 & 18 & 20 & \end{array}$

Responsável pelo sustento

$\begin{array}{lccccccc}\text { Pai e mãe } & 106 & 57,9 & 57 & 53,8 & 49 & 46,2 & 0,55 \\ \text { Pai ou mãe/outros } & 77 & 42,1 & 38 & 49,4 & 39 & 50,6 & \end{array}$

Escolaridade do pai

Ensino fundamental

completo ou +

Ensino fundamental

incompleto

Escolaridade da mãe

Ensino fundamental

completo ou +

Ensino fundamental incompleto

77

116

$63,4 \quad 64$

$55,2 \quad 52 \quad 44,8$

67

$36,6 \quad 31$

$46,3 \quad 36$

53,7

0,24

Faltou à escola por dor de dente

Não

Sim

5

131

71,6

67

51,1

64

48,9

52

28,4

28

$53,8 \quad 24$

46,2

Foi ao dentista alguma vez

Sim

Não

122

61

66

$97,3 \quad 94$

52,8

84

47,2

0,14

Há quanto tempo foi ao dentista

Até 2 anos

2 anos ou +

Nunca foi

14

14

$58,9 \quad 49$

60

33,3

35

Particular ou outros

Público

87

28,

71,3

2,7

1

20

4

80

10 
Satisfação com o atendimento

$\begin{array}{lccccccc}\text { Bom/ótimo } & 103 & 56,3 & 49 & 47,6 & 54 & 52,4 & 0,18 \\ \text { Ruim/regular } & 80 & 43,7 & 46 & 57,5 & 34 & 42,5 & \end{array}$

Motivo da procura pelo atendimento

\begin{tabular}{lccccccc} 
Rotina & 135 & 78,9 & 81 & 60,0 & 54 & 40,0 & 0,001 \\
Dor & 11 & 6,4 & 4 & 36,4 & 7 & 63,6 & \\
Tratamento & 25 & 14,6 & 5 & 20,0 & 20 & 80,0 & \\
familiar & & & & & & & \\
Até 2 salários-mínimos & 5 & 3,3 & 2 & 40,0 & 3 & 60,0 & 0,617 \\
+ que 2 salários-mínimos & 146 & 96,7 & 75 & 51,4 & 74 & 50,6 & \\
\hline
\end{tabular}

Fonte: Elaboração própria (2010).

Tabela 3. Análise de regressão de Poisson ajustada para estimar o efeito das características sociodemográficas e comportamentais nos componentes cariado e restaurado em crianças de 5 anos. Lindóia, Águas de Lindóia e Monte Alegre do Sul, 2010.

\begin{tabular}{|c|c|c|}
\hline \multirow{5}{*}{ VARIÁVEL } & COMPONENTE & COMPONENTE CARIADO \\
\hline & RESTAURADO & Águas de Lindóia/Monte \\
\hline & Lindóia & Alegre do Sul \\
\hline & Análise ajustada & Análise ajustada \\
\hline & PR (IC 95\%) & PR (IC 95\%) \\
\hline Escolaridade da mãe & $p=0,01$ & - \\
\hline Ensino fundamental incompleto & $1,599(1,119-2,285)$ & - \\
\hline Ensino fundamental completo ou + & 1 & - \\
\hline Foi ao dentista alguma vez na vida & $p=0,002$ & $p=0,000$ \\
\hline Sim & 1 & 1 \\
\hline Não & $0,386(0,208-0,714)$ & $1,975(1,510-2,584)$ \\
\hline Onde foi ao dentista & - & $p=0,052$ \\
\hline Particular ou outros & - & 1 \\
\hline Público & - & $1,644(0,996-2,714)$ \\
\hline Motivo da procura pelo atendimento & - & $p=0,003$ \\
\hline Rotina & - & 1 \\
\hline Dor & - & $1,343(0,783-2,303)$ \\
\hline Tratamento & - & $1,673(1,196-2,341)$ \\
\hline
\end{tabular}

Fonte: Elaboração própria (2010). 


\section{Discussão}

Compreender a epidemiologia da saúde bucal infantil e seu comportamento é importante na identificação de prováveis oportunidades de diminuir a prevalência da doença na população no futuro. ${ }^{1,10,18,19}$ A percepção que se aflorou, após as análises realizadas neste estudo, foi que mesmo em municípios de proximidade geográfica, com número de habitantes menor que 20 mil e com fluoretação das águas de abastecimento, a realidade da saúde bucal dessas crianças é distinta. No censo demográfico realizado em

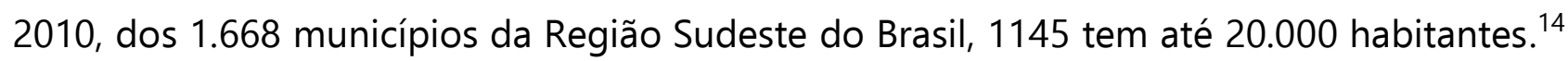
Apesar disso, não são comuns levantamentos epidemiológicos de saúde bucal envolvendo municípios de pequeno porte populacional, o que aumenta a importância deste estudo. Esses dados reforçam a importância da descentralização das ações em saúde na atenção primária e das decisões tomadas o mais próximo possível da comunidade que será beneficiada.

As médias do índice ceod aos cinco anos dos três municípios corroboraram com os resultados do levantamento nacional de saúde bucal do Brasil em 2010, ${ }^{4}$ o qual apresentou ceod de 2,23 para o interior da região Sudeste do Brasil, onde se localizam os municípios deste estudo. No entanto, diferiram de outros estudos locais nesta mesma faixa etária: Pelotas (RS) ${ }^{20}$ apresentou ceod de 1,9 em 2010, Montes Claros (MG) ${ }^{21}$ de 1,7 em 2009 e Cascavel $(P R)^{22}$ de 2,42 em 2010. Em outro estudo, com população de até 10 mil habitantes, na região sudeste do estado de São Paulo, o ceod de crianças de 5 anos foi de 3,44. ${ }^{23}$

No entanto, a situação real da saúde bucal não se verifica apenas pela média da experiência de cárie numa determinada população, é necessário conhecer também a distribuição da doença e em que estágio ela se encontra. ${ }^{2,8}$ Neste estudo, esta análise foi realizada pelo cálculo do SIC Index, que apresentou comportamento semelhante ao ceod em todos os municípios do nosso estudo e também pela análise dos componentes destes índices, que revelaram a existência de desigualdade na distribuição da experiência de cárie. 
O município de Lindóia, com a maior média de ceod e SIC entre os três municípios, apresentou a maior parte (> que $70 \%$ ) dos componentes restaurados, sendo que no grupo de baixa experiência de cárie nenhuma criança necessitava de tratamento restaurador. Porém, no grupo de alta experiência da doença, 28,1\% apresentaram um ou mais dentes com necessidade de tratamento. Esses resultados demonstraram que apesar da alta cobertura de tratamento odontológico, existe diferença no acesso a ele entre as crianças deste município. Segundo Gillcrist et al., ${ }^{19}$ crianças de famílias com menores rendas têm menor acesso aos serviços de saúde, no entanto, no nosso estudo esta hipótese não se confirmou.

A água de abastecimento de Lindóia é fluoretada desde 2005, portanto, a população estudada está coberta pelo método preventivo desde o nascimento e, mesmo assim, mais de $50 \%$ apresentou experiência de cárie. Uma hipótese levantada para esse resultado é que a água fluoretada não deve atingir toda a população e, segundo dados da Secretaria de Planejamento e Desenvolvimento Regional - SEADE, em 2010 aproximadamente 87\% desta possuía esse benefício no município. ${ }^{15}$ Neste caso, onde a água fluoretada não alcança toda a população de um mesmo município, ela passa a ser um fator de causa de iniquidade, pois contribui para aumento das diferenças sociais em saúde. ${ }^{24}$

De acordo com Mello et al., $^{23}$ a água tratada não chega justamente naquela população com maior necessidade de cuidado. Esta observação sugere que a água fluoretada não vem beneficiando a população de modo homogêneo e que há espaço para uma redução mais intensa dos indicadores epidemiológicos da cárie no país através da ampliação de cobertura do sistema de abastecimento de água nas cidades que já disponibilizam o benefício.

Os dois outros municípios estudados apresentaram dados totalmente inversos à Lindóia. Apesar de ambos terem a população estudada coberta pela fluoretação das águas de abastecimento desde o nascimento e apresentarem a média dos índices ceod e SIC menores, a maior parte das crianças com experiência de cárie não teve acesso ao tratamento 
odontológico, sem diferença entre os grupos de baixa e alta experiência com relação à porcentagem de dentes tratados. No município de Águas de Lindóia, 87\% das crianças apresentaram dentes com lesões de cárie não tratada, diferente de outro estudo, ${ }^{25}$ no qual essa porcentagem foi em torno de $45 \%$.

Junqueira et al. ${ }^{25}$ comparou o acesso ao tratamento em diferentes faixas etárias no município de São Paulo e concluiu que a idade de 5 anos foi a que teve pior acesso. É válido ressaltar que a utilização dos serviços de saúde está relacionada às formas de financiamento, serviços e recursos disponíveis para a população, mas também está relacionada às necessidades e ao comportamento individual em relação aos problemas de saúde. ${ }^{12}$ Nas crianças, está relacionada ao comportamento dos pais ou responsáveis, visto que nesta fase da vida existe uma dependência destes para a o uso de serviços odontológicos. 11,13,20

Dentre os fatores associados ao acesso ou ao tratamento restaurador, a variável "ter ido ao dentista alguma vez" se mostrou associada tanto na análise do componente restaurado em Lindóia como do componente cariado nos outros dois municípios, porém no sentido inverso. Está claro que as crianças com dentes restaurados necessariamente precisam ter ido ao dentista, e foi o que análise demonstrou para o município de Lindóia. Por outro lado, nos municípios com maior porcentagem de dentes cariados o fato de ter ido ao dentista se apresentou como fator de proteção, ou seja, as crianças com acesso ao dentista apresentavam 1,9 vezes mais chance de não ter dentes cariados. Esses dados preocupam, pois estudos demonstram que a prevalência de cárie aumenta com a idade 4,26,27 e a dificuldade no acesso à consulta odontológica na infância pode gerar um agravamento na carga de doença, o que ocasiona a necessidade de extração dentária no futuro. ${ }^{25,26}$ Em Águas de Lindóia, apesar de 49,6\% das crianças se apresentarem livres de cárie, a maior parte daquelas com experiência da doença apresentaram dentes não tratados.

Além do fato de ter ido ao dentista se apresentar associado ao tratamento restaurador em Lindóia, a criança cuja mãe não completou o Ensino Fundamental apresentou maior risco de ter dentes restaurados. Esses dados diferem dos resultados de 
Goettems et al., ${ }^{28}$ que demonstraram que os filhos de mães com baixa escolaridade não visitavam o dentista regularmente ou tinham mais risco de não receber tratamento odontológico. No nosso estudo, 40,9\% das crianças desse município eram livres de cárie e entre as demais, com experiência de cárie, 82,3\% tinham os dentes restaurados, o que significa que as crianças cujas mães tinham maior grau de escolaridade tiveram menor experiência de cárie e, portanto, menor necessidade de tratamento restaurador.

A associação encontrada entre o uso de serviços odontológicos na faixa etária de cinco anos e o grau de escolaridade da mãe, em Lindóia, destaca a importância da educação em saúde, no sentido de conscientizar os pais quanto ao uso de serviços odontológicos em caráter preventivo e promocional, e não apenas terapêutico. Hamati et al. ${ }^{12}$ destaca que, quanto menores o nível educacional e o vínculo das mães com uma Unidade de Saúde da Família - USF que possui equipe de saúde bucal - ESB, maior será o uso de procedimentos restauradores por seus filhos. Os pais são modelos para os filhos e são responsáveis pelos cuidados diários da criança, bem como pela tomada de decisões acerca da mesma e pelo uso dos serviços odontológicos. ${ }^{11,13,27,28}$ A saúde das crianças, como parte da Política Nacional de Atenção Básica, deve ser tratada com atenção especial em relação ao crescimento e desenvolvimento das mesmas e prevenção de agravos em saúde. ${ }^{29}$

\section{Conclusão}

Os resultados deste estudo sugerem que ter realizado tratamento odontológico ou não, nesta população de cinco anos, esteve associado à procura dos serviços de saúde oferecidos nestes municípios de pequeno porte, sendo que o grau de escolaridade da mãe pode influenciar na saúde bucal dos filhos. O estabelecimento de políticas públicas de promoção de saúde e reorientação de serviços que facilitem a utilização dos serviços odontológicos e possibilitem o atendimento às crianças de pouca idade nestes municípios é necessário. 


\section{Referências}

1. Kilpatrick NM, Neumann A, Lucas N, Chapman J, Nicholson JM. Oral health inequalities in a national sample of Australian children aged 2-3 and 6-7 years. Austr Dent J. 2012;57(1):38-44. https://doi.org/10.1111/j.1834-7819.2011.01644.x.

2. Fernández $C N$, Squassi $A$, Bordoni N. Dental status and dental treatment demands in preschoolers from urban and underprivileged urban areas in Mendoza city, Argentina. Acta Odontol Latinoam. 2015;28(1):13-21. https://doi.org/10.1590/S185248342015000100002 .

3. Brasil. Ministério da Saúde. Secretaria de Atenção à Saúde. Departamento de Atenção Básica. Projeto SB Brasil 2003: condições de saúde bucal da população brasileira 20022003. Resultados principais. Brasília, DF: MS; 2004.

4. Brasil. Ministério da Saúde. Projeto SB Brasil 2010: pesquisa nacional de saúde bucal 2010. Resultados principais. Brasília, DF: Ministério da Saúde; 2011.

5. Pinheiro RS, Torres TZG. Uso de serviços odontológicos entre os Estados do Brasil. Cienc Saude Colet. 2006;11(4):999-1010. http://dx.doi.org/10.1590/S141381232006000400021.

6. Antunes JLF, Narvai PC. Políticas de saúde de bucal no Brasil e seu impacto sobre as desigualdades em saúde. Rev Saude Publica 2010;44(2):360-5. http://dx.doi.org/10.1590/S0034-89102010005000002.

7. Instituto Brasileiro de Geografia e Estatística. Pesquisa nacional por amostra de domicílio: um panorama da saúde no Brasil: acesso e utilização dos serviços, condições de saúde e fatores de risco e proteção à saúde. Rio de Janeiro: IBGE, 2010. 256 p.

8. Ferreira SH, Beria JU, Kramer PF, Gerson Feldens E, Feldens CA. Dental caries in 0- to 5year-old Brazilian children: prevalence, severity and associated factors. Int J Paediatr Dent. 2007;17(4):289-96. http://dx.doi.org/10.1111/j.1365-263X.2007.00831.x

9. Bonanato K, Pordeus IA, Moura-Leite FR, Ramos-Jorge ML, Vale MP, Paiva SM. Oral disease and social class in a random sample of five-year-old preschool children in a Brazilian city. Oral Health Prev Dent. 2010;8(2):125-32. http://dx.doi.org/10.3290/j.ohpd.a19202

10. Catani D, Meirelles MPR, Sousa ML. Cárie dentária e determinantes sociais de saúde em escolares do município de Piracicaba - SP. Rev Odontol UNESP. 2010;39(6):344-350. 
11. Hooley M, Skouteris H, Boganin C, Satur J, Kilpatrick N. Parental influence and the development of dental caries in children aged 0-6 years: A systematic review of the literature. J Dent. 2012;40(11):873-85. http://dx.doi.org/10.1016/j.jdent.2012.07.013.

12. Hamati F, Rocha JS, Baldani MH. Prevalência de cárie, dor e uso de serviços odontológicos por crianças em áreas com e sem Equipes de Saúde Bucal na Estratégia Saúde da Família no município de Ponta Grossa, Paraná, Brasil. Rev Bras Pesqui Saude. 2014;16(3):48-57. https://doi.org/10.21722/rbps.v16i3.13908

13. Castilho AR, Mialhe FL, Barbosa Tde S, Puppin-Rontani RM. Influence of family environment on children's oral health: a systematic review. J Pediatr (Rio J). 2013;89(2):116-23. https://doi.org/10.1016/j.jped.2013.03.014.

14. Instituto Brasileiro de Geografia e Estatística. Pesquisa de Informações Básicas Municipais 2010 [acesso 2016 ago 1]. Disponível em: http://www.ibge.gov.br/home/estatistica/populacao/censo2010/indicadores_sociais_mu nicipais/indicadores_sociais_municipais.pdf.

15. Fundação Seade. Perfil dos municípios paulistas. c2018 [acesso 2015 ago. 1]. Disponível em http://www.perfil.seade.gov.br/.

16. Secretaria de Estado de Saúde de São Paulo (SES-SP). Condições de Saúde Bucal no Estado de São Paulo em 2002. São Paulo: SES-SP; 2002.

17. World Health Organization. Bucal health surveys, basics methods. 4th ed. Geneva: WHO; 1997.

18. Borges HC, Garbín CAS, Saliba O, Saliba NA, Moimaz SAS. Socio-behavioral factors influence prevalence and severity of dental caries in children with primary dentition. Braz Oral Res. 2012;26(6): 564-570. http://dx.doi.org/10.1590/S180683242012000600013.

19. Gillcrist J, Brumley DE, Blackford JU. Community socioeconomic status and children's dental health. J Am Dent Assoc. 2001;132(2):216-22. https://doi.org/10.14219/jada.archive.2001.0158

20. Camargo MBJ, Barros AJD, Frazão P, Matijasevic A, Santos IS, Peres MA et al. Predictors of dental visits for routine check-ups and for the resolution of problems among preschool children. Rev Saude Publica. 2012;46(1):87-97. http://dx.doi.org/10.1590/S0034-89102012005000004. 
21. Palma ABO, Martins AMEBL, Ferreira RC, Mendes LA, Cachoeira NL, Borges CFN et al. Saúde bucal de crianças de 5 anos de idade de Montes Claros, Brasil. Unimontes Cientificas. 2012;14(1):80-7.

22. Berti M, Furlanetto DLC, Walker MMS, Baltazar MMM, Bianchi FJ. Levantamento epidemiológico de cárie dentária em escolares de 5 e 12 anos de idade do município de Cascavel, PR. Cad Saude Colet. 2013;21(4):403-6. http://dx.doi.org/10.1590/S1414$462 \times 2013000400007$.

23. Mello TRC, Antunes JLF, Waldman EA. Prevalência de cárie não tratada na dentição decídua em áreas urbanas e rurais do Estado de São Paulo, Brasil. Rev Panam Salud Publica. 2008;23(2):78-84.

24. Meirelles MPR, Sousa MLR. A importância da fluoretação da água de abastecimento em municípios de pequeno porte. Rev Fac Odontol Porto Alegre. 2005; 46(2):15-19.

25. Junqueira SR, Frias AC, Zilbovicius C, Araujo ME. Oral health and access to dental care services in relation to the Health Necessities Index. Cien Saude Colet. 2012;17(4):101524. http://dx.doi.org/10.1590/S1413-81232012000400023.

26. Gushi LL, Rihs LB, Soares MC, Forni TIF, Vieira V, Wada RS et al. Cárie dentária e necessidades de tratamento em adolescentes do Estado de São Paulo, 1998 e 2002. Rev Saude Publica. 2008;42(3)480-6. http://dx.doi.org/10.1590/S0034-89102008005000015.

27. Rodrigues LAM, Martins AMEBL, Silveira MF, Ferreira RC, Souza JGS, Silva JM et al. Uso de serviços odontológicos entre pré-escolares: estudo de base populacional. Cienc Saude Colet. 2014;19(10):4247-56. http://dx.doi.org/10.1590/1413812320141910.13382013.

28. Goettems ML, Ardenghi TM, Demarco FF, Romano AR, Torriani DD. Children's use of dental services: Influence of maternal dental anxiety, attendance pattern, and perception of children's quality of life. Community Dent Oral Epidemiol. 2012 Out;40(5):451-8. http://dx.doi.org/10.1111/j.1600-0528.2012.00694.x

29. Ribeiro LCC, Paula FA, Leite MB, Barbosa, BR. As orientações recebidas na estratégia de saúde da família e outros serviços de saúde no âmbito da saúde. J Manag Prim Health Care. 2015;6(1):36-45. 


\section{Minicurrículo}

Maria Paula Rando-Meirelles | ORCiD: 0000-0001-6197-1757

Doutora em Odontologia, com área de concentração em Saúde Coletiva pela FOP/UNICAMP.

Professora do curso de Odontologia da Universidade Paulista - UNIP, Campinas, São Paulo, Brasil.

Vinícius Aguiar Lages | ORCiD: 0000-0003-0110-3987

Doutor em Odontologia, com área de concentração em Saúde Coletiva, pela FOP/UNICAMP.

Professor do curso de Odontologia da Faculdade Maurício de Nassau - UNINASSAU, Teresina, Piauí, Brasil.

Marília Jesus Batista | ORCiD: 0000-0002-0379-3742

Doutora em Odontologia, com área de concentração em Saúde Coletiva, pela FOP/UNICAMP.

Professora da Faculdade de Medicina de Jundiaí - FMJ, Jundiaí, São Paulo, Brasil.

\section{Silvia Cypriano}

Doutora em Odontologia, com área de concentração em Saúde Coletiva, pela FOP/UNICAMP.

Professora do curso de Odontologia da Pontifícia Universidade Católica de Campinas - PUC/Campinas,

Campinas, São Paulo, Brasil.

Maria da Luz Rosário de Sousa | ORCiD: 0000-0002-0346-5060

Doutora em Saúde Pública pela Universidade de São Paulo/USP

Professora Titular do Departamento de Odontologia Social da FOP/UNICAMP, Piracicaba, São Paulo, Brasil. 